\title{
COMUNICAÇÃO
}

\section{AVALIAÇÃO TÉCNICA DE UM ASPERSOR CANHÃO DO TIPO TURBINA ${ }^{1}$}

\author{
Technical evaluation of a gun sprinkler of the turbine type
}

\author{
Júlio H. Fernandes César ${ }^{2}$, Antônio Marciano da Silva ${ }^{3}$, \\ Felizardo Adenilson Rocha ${ }^{4}$, Carlos Rogério de Mello
}

\begin{abstract}
RESUMO
Objetivou-se com este trabalho avaliar o desempenho técnico do aspersor canhão do tipo turbina, modelo "Mercury", fabricado pela Società Hidromecânica Emiliana (SIME) na Itália e importado pela indústria Metal Lavras Ltda. Para este estudo, foram testados três bocais (16, 20 e $22 \mathrm{~mm})$, com pressões de serviço de 250, 300, 350, 400, 450 e $500 \mathrm{kPa}$, pelo método de ensaio radial, com a finalidade de verificar a influência dessas variáveis na uniformidade de distribuição, intensidade de aplicação, diâmetro médio das gotas, raio de alcance e uniformidade de rotação. Tabelas contendo os valores de intensidade média de precipitação, espaçamentos recomendados e coeficientes de uniformidade foram criadas, cobrindo uma deficiência demonstrada no catálogo do fabricante. Observou-se que o diâmetro médio das gotas aumentou à medida que se reduziu a pressão de serviço, e o comportamento do mesmo não se adapta ao modelo clássico de aumento gradual do diâmetro médio das gotas, pois foram constatados vários diâmetros de gotas ao longo do raio molhado. A equação potencial ajustou-se bem aos dados de vazão versus pressão, e o bocal $16 \mathrm{~mm}$ mostrou-se mais susceptível às variações de pressão de serviço. O perfil de distribuição demonstrado é do tipo "doughnut" e retangular. Os dados de uniformidade de rotação demonstraram a eficiência do sistema de rotação do tipo turbina. Pelas tabelas geradas, recomenda-se o uso delas em substituição ao catálogo do fabricante.
\end{abstract}

Termos para indexação: Aspersor canhão, turbina, avaliação.

\section{ABSTRACT}

This work intended to evaluate the technical performance of a gun sprinkler of the type turbine model "Mercury" manufactured by Società Hidromecânica Emiliana (SIME) in Italy and imported by the Brazilian company Metal Lavras Ltda. The study was developed with three nozzles (16, 20 and $22 \mathrm{~mm}$ ), with service pressures of 250 , $300,350,400,450$ and $500 \mathrm{kPa}$ by the radial method test, with the purpose of verifying the influence of these parameters on distribution uniformity, application intensity, medium diameter of drops, reach radius and rotation uniformity. Tables with values of average precipitation intensity, recommended spacing and values of uniformity coefficient were created, overcoming a deficiency demonstrated by manufacturer's catalog. It was verified that the medium diameter of drops increased with the decrease of the service pressure, and its behavior did not adapt to the classic model of gradual increase of the medium diameter of drops because several diameters were verified along of the wet radius. The potential equation was adjusted to the data of discharge versus pressure, with the nozzle of $16 \mathrm{~mm}$ being the most susceptible to the variations of service pressure. The water distribution profile followed the type "doughnut" and rectangular. The data of rotation uniformity demonstrated the efficiency of the rotation system of the turbine type. It was recommended the use of the tables produced, in substitution to the manufacturer's catalog.

Index terms: Gun sprinkler, turbine, evaluation.

(Recebido para publicação em 31 de julho de 2001 e aprovado em 26 de fevereiro de 2003)

\footnotetext{
1. Parte da Dissertação de Mestrado do primeiro autor apresentada àUNIVERSIDADE FEDERAL DE LAVRAS/UFLA - Caixa Postal 37 - $37200-000$ - Lavras, MG, para obtenção do grau de Mestre.

2. Engenheiro Agrícola, M.Sc em Engenharia Agrícola, Faculdades da Terra de Brasília, Brasília, DF.

3. Engenheiro Agrônomo, Dr., Professor Titular do Departamento de Engenharia/UFLA, Pesquisador Bolsista do CNPq

4. Engenheiro Agrícola, M.Sc. em Engenharia Agrícola - Irrigação e Drenagem.

5. Engenheiro Agrícola, Dr., Departamento de Engenharia/UFLA, rog.mello@terra.com.br
} 
A irrigação por aspersão tem contribuído acentuadamente para o aumento da área irrigada e do número de empresas fabricantes de equipamentos de irrigação no Brasil; entretanto, são poucas as empresas que mantêm uma área de desenvolvimento do produto com controles técnicos de qualidade e campos de testes de desempenho.

$\mathrm{O}$ aspersor canhão do tipo turbina tem sua rotação originada pela reação do jato em contato com as palhetas da turbina, obtendo uma rotação uniforme e dispondo de um sistema de reversão lenta e gradual, diferenciando-se dos aspersores tradicionais, amplamente difundidos (TOMAZELA, 1996).

Nos tempos de elevada competitividade entre os fabricantes nacionais, incrementada pela concorrência externa, deflagrada pela globalização da economia e abertura do mercado às importações, acentua-se a necessidade de uma melhoria na qualidade dos produtos nacionais, a qual tem, na avaliação dos produtos, um subsídio de extrema valia (KELLER e BLIESNER, 1990; BERNARDO, 1995). A aplicação de testes segundo as normas padronizadas torna-se, cada vez mais, uma necessidade de sobrevivência no mercado.

A adaptabilidade do produto importado às condições brasileiras é, sem dúvida, um requisito essencial para o sucesso comercial e para a manutenção da confiança dos consumidores em relação a um importador. Essa premissa é atingida com a sujeição do produto aos testes propostos pela norma ISO 7749-2 (ISO, 1990), em que os possíveis defeitos ou qualidades são apontados. Cria-se, assim, subsídios para que o importador obtenha, em futuras importações, uma garantia maior de qualidade do produto junto ao fabricante; trazendo para si um retorno seguro do capital investido (MARTINBENITO et al., 1992; ROCHA, 2000).

Assim sendo, desenvolveu-se o presente trabalho com a finalidade de avaliar o desempenho técnico de aspersores canhão do tipo turbina que a empresa Metal Lavras Ltda. importa da Società Hidromecânica Emiliana (SIME) Itália.

Os ensaios foram realizados no Campo de Testes da Indústria Metal Lavras, situada na BR 265, km 142, município de Lavras, região sul de Minas Gerais, geograficamente definida pelas coordenadas de $21^{\circ} 14^{\prime}$ de latitude Sul, $45^{\circ} 00^{\prime}$ de longitude Oeste e altitude de $910 \mathrm{~m}$.

Para a realização dos ensaios, utilizou-se um aspersor da SIME (Società Hidromecânica Emiliana) importado da Itália pela indústria Metal Lavras Ltda. O modelo testado foi o MERCURY, com diâmetro de base $50,8 \mathrm{~mm}$ e bocais de 16,20 e $22 \mathrm{~mm}$, sendo as pressões estabelecidas dentro da faixa de operação recomendada pelo fabricante. O método de ensaio utilizado foi o preconizado pela norma ISO 7749-2 (ISO, 1990).

A área para testes foi de $22000 \mathrm{~m}^{2}$, plana, com reservatório de $40 \mathrm{~m}^{3}$, abastecido por um conjunto motobomba diesel com motor MWM 6-10 T e bomba FAL com capacidade de $125 \mathrm{~m}^{3} / \mathrm{h}$ e elevação de 100 m.c.a., situada no ribeirão Água Limpa, próximo à indústria. Do reservatório ao equipamento, outro conjunto motobomba foi utilizado para atender aos testes. $\mathrm{O}$ aspersor foi instalado a uma altura de $1,70 \mathrm{~m}$ em tripé com tubo de subida de 76,2 mm, fabricado para uso em campo. A altura do bocal em relação ao solo foi de $2,10 \mathrm{~m}$.

A vazão do aspersor foi medida por meio de um medidor de palhetas Ômega previamente aferido, com a média de três leituras por teste. Com os valores de pressão e vazão, a equação potencial foi aplicada para representar a relação vazão versus pressão (KELLER e BLIESNER, 1990).

A velocidade de rotação foi medida por cronômetro, totalizando o tempo necessário para uma volta completa e por quadrante. A medição por quadrante foi necessária para comprovar a uniformidade de rotação e a eficiência do sistema mecânico, tipo turbina. Foram realizados três tomadas de tempo por teste.

A distribuição dos coletores na área foi na forma radial, de acordo com a norma ISO 7749-2 (ISO, 1990). O espaçamento entre coletores foi de $2 \mathrm{~m}$, conforme especificado pela norma para aspersores com diâmetro de cobertura maior que $20 \mathrm{~m}$. A altura da borda do coletor em relação ao solo foi de $0,70 \mathrm{~m}$ e do centro do bocal em relação à borda do coletor de 1,40 m, atendendo à norma ISO 7749-2 (ISO, 1990), que estabelece uma altura de dez vezes o diâmetro do tubo de subida e não menos que $0,50 \mathrm{~m}$. Os testes tiveram duração de uma hora, conforme especifica a norma citada. A necessidade da total ausência do vento exigida pela norma foi contornada com a realização dos testes nos períodos do amanhecer e entardecer.

Quanto às perdas por evaporação, foram utilizados três coletores próximos à área de teste. A perda foi estimada pela média dos coletores e reposta em cada coletor. Valores de temperatura, umidade e velocidade do vento foram obtidos de uma estação climatológica Davis.

Os coeficientes de uniformidade foram determinados com a simulação de um conjunto de aspersores operando simultaneamente em disposições retangulares e triangulares, com espaçamentos inferiores a $60 \%$ do diâmetro molhado pelo aspersor, sendo considerado o raio de alcance como a distância do aspersor ao coletor 
de precipitação mínima de $0,25 \mathrm{~mm} / \mathrm{h}$, conforme a norma ISO 7749-2 (ISO, 1990). Se a lâmina requerida estiver entre dois coletores, o raio será obtido por interpolação linear, conforme a norma ISO 7749-2 (ISO, 1990).

O programa computacional CACHT3D, versão 4.6, foi utilizado para simular uma malha de coletores e aspersores dispostos de forma retangular e triangular com espaçamentos variados e inferiores a $60 \%$ do diâmetro molhado (ROCHA, 2000). Os coeficientes de uniformidade de Christiansen também foram gerados para cada espaçamento.

As intensidades de precipitações para cada tipo de disposição foram calculadas seguindo as orientações de Gomes (1994), como a seguir:

Disposição Retangular

$$
I=\frac{q}{S_{1} \cdot S_{2}}
$$

Disposição Triangular

$$
\mathrm{I}=\frac{\sqrt{3} \cdot \mathrm{q}}{1.5 \cdot \mathrm{S}_{1}^{2}}
$$

em que $\mathrm{I}=$ intensidade média de aplicação $\left(\mathrm{mm} \mathrm{h}^{-1}\right) ; \mathrm{q}$ = vazão do aspersor $\left(\mathrm{Lh}^{-1}\right) ; \mathrm{S}_{1}=$ espaçamento dentro da linha $(\mathrm{m}) ; \mathrm{S}_{2}=$ espaçamento entre linhas $(\mathrm{m})$.

Segundo Vilela (1995), o diâmetro médio das gotas pode ser calculado em função do peso médio do grânulo pela equação:

$$
\mathrm{D}=1,0874 \cdot \mathrm{P}^{0,364412}
$$

em que $\mathrm{D}=$ diâmetro médio das gotas $(\mathrm{mm})$ e $\mathrm{P}=$ peso médio do grânulo (mg).

Para o aspersor Mercury, no teste de diâmetro médio das gotas, foram avaliados três valores de pressão para os três bocais selecionados com as mesmas características de montagem para o teste de uniformidade descrito anteriormente.

O método baseia-se no método da farinha, em que essa é acondicionada em recipientes de vidro com $0,150 \mathrm{~m}$ de diâmetro e $0,04 \mathrm{~m}$ de altura, alinhadas e espaçadas de 4 em $4 \mathrm{~m}$ em relação ao centro do aspersor, para o bocal de $16 \mathrm{~mm}$; de $5 \mathrm{em} 5 \mathrm{~m}$ para o bocal de $20 \mathrm{~mm}$ e de 7 em $7 \mathrm{~m}$ para o bocal de $22 \mathrm{~mm}$. Os recipientes foram colocados sobre suportes de madeira a $0,70 \mathrm{~m}$ do solo, para evitar respingos (VILELA, 1995; ROCHA, 1998; ROCHA 2000).

Na Figura 1 são apresentadas as equações potenciais ajustadas e os respectivos coeficientes de determinação. Para cada modelo e bocal, têm-se cinco valores de pressão e vazão.

A equação potencial ajustou-se muito bem aos dados. Um maior expoente na equação potencial permite uma maior variação da vazão em função de uma variação mínima da pressão de serviço. Sendo assim, pode-se afirmar que o bocal de $16 \mathrm{~mm}$ está mais susceptível às variações de pressão, e o bocal de $20 \mathrm{~mm}$ permite uma oscilação maior na pressão de serviço, sem causar grande variação da vazão.

Nas Figuras 2 e 3 são apresentados alguns dos perfis de distribuição das intensidades de precipitação para o modelo Mercury, de acordo com o bocal e a pressão estabelecidos, gerados por este trabalho.

Em todos bocais observados, tem-se uma mudança de perfil de distribuição de uma pior situação para uma melhor, à medida que se aumenta a pressão de serviço.

Aspersores de grande porte trabalhando com baixa pressão de serviço apresentam uma quebra do jato deficiente e, conseqüentemente gotas de maior diâmetro e uma precipitação maior na extremidade final do raio molhado. Dessa forma, foi caracterizada, para o bocal de $22 \mathrm{~mm}$ de diâmetro interno trabalhando a baixas pressões de serviço, a formação de perfil de distribuição de água do tipo "doughnut".

Na Figura 4 são apresentados valores de rotação por bocal e pressão. Nessa Figura, observa-se um aumento uniforme da rotação à medida que aumentam as pressões de serviço e para os bocais de diâmetros maiores, a rotação tende a ser mais uniforme. 


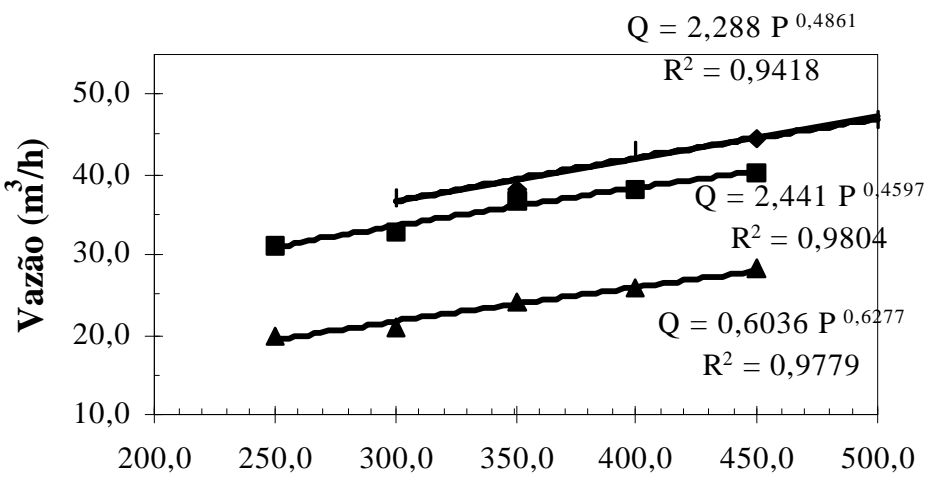

Pressão ( kPa )

I Bocal $22 \mathrm{~mm}$ Bocal $20 \mathrm{~mm} \boldsymbol{\Delta}$ Bocal $16 \mathrm{~mm}$

FIGURA 1 - Dados comparativos da equação característica da vazão em função da pressão para o aspersor Mercury, bocais 16,20 e $22 \mathrm{~mm}$.

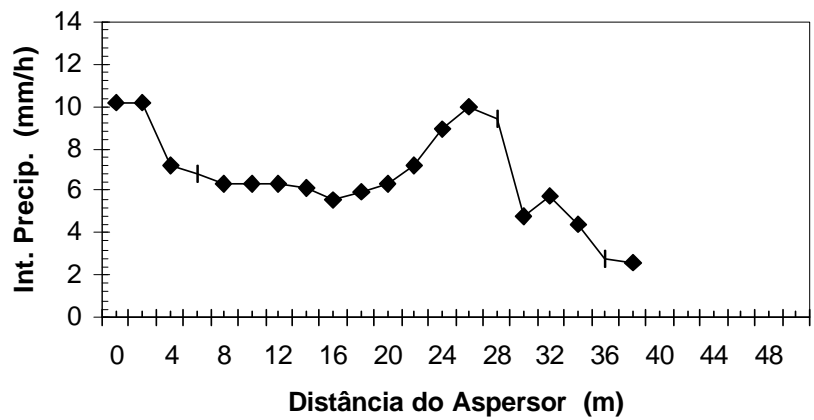

FIGURA 2 - Perfil de distribuição da intensidade de precipitação em função da distância do aspersor para o aspersor Mercury com bocal de $22 \mathrm{~mm}$ e pressão de $300 \mathrm{kPa}$.

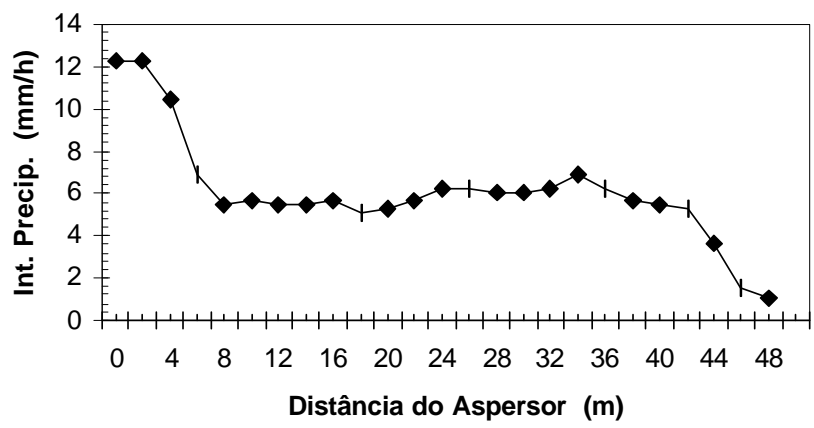

FIGURA 3 - Perfil de distribuição da intensidade de precipitação em função da distância do aspersor para o aspersor Mercury com bocal de $22 \mathrm{~mm}$ e pressão de $500 \mathrm{kPa}$. 


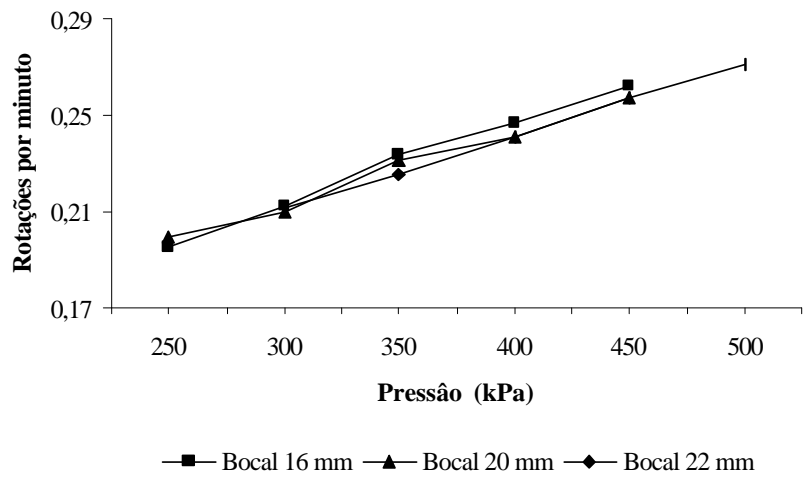

FIGURA 4 - Velocidade de rotação do aspersor Mercury, em função da pressão e do diâmetro interno do bocal.

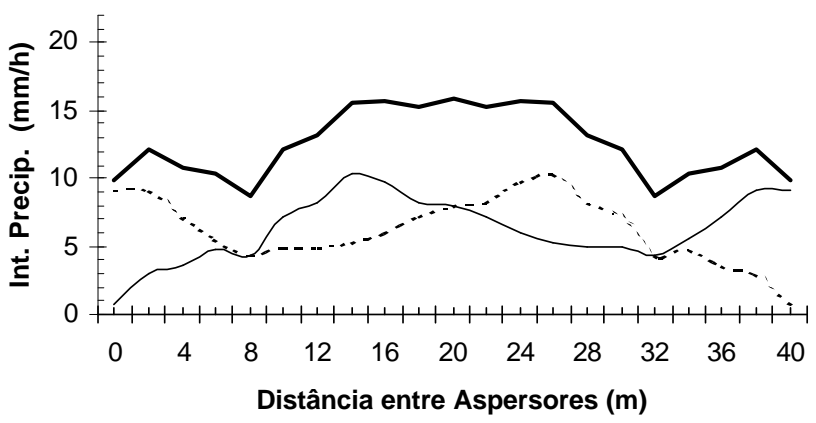

FIGURA 5 - Sobreposição para aspersores com perfil do tipo "doughnut", espaçados de 40 m.

$\mathrm{O}$ aspersor Mercury apresentou, em todos os ensaios, rotação inferior à rotação máxima de 3,0 rpm, preconizada pela norma ISO 7749-1 (ISO, 1995).

Os resultados dos coeficientes de uniformidade de Cristhiansen para as disposições retangulares e triangulares para diferentes espaçamentos estão apresentados nas Tabelas 1 e 2, na qual também pode-se ver os valores de intensidade média de precipitação.

Para a disposição retangular, o maior espaçamento considerado foi entre linhas, enquanto na disposição triangular, foi dentro da linha de aspersores.

A formação de perfis do tipo "doughnut" para aspersores de grande porte produz aspectos interessantes na sobreposição dos mesmos. Observa-se na Tabela 3, para a disposição triangular, que os valores de CUC não seguem um comportamento regular, ou seja, são comuns valores de coeficiente de uniformidade alternarem ora acima de $80 \%$, ora abaixo. Com isso, a tabela apresenta uma alternância de espaçamentos recomendados e não-recomendados.

Nas Figuras 5 e 6 observa-se o perfil resultante da sobreposição de perfis. Verifica-se uma melhor distribuição quando áreas com maior precipitação sobrepõem áreas de menor precipitação (Figura 5) e de uma distribuição irregular, com CUC abaixo de $80 \%$, quando altos valores de precipitação são somados (Figura 6).

Os resultados em relação ao diâmetro médio das gotas produzidas pelo aspersor "Mercury" para três valores de pressão de serviço e três bocais são apresentados nas Figuras 7, 8 e 9. Constata-se que, de maneira geral, o aumento na pressão de serviço provoca a diminuição dos diâmetros médios das gotas, estando de acordo com o afirmado por Carvalho (1991). No entanto, pode-se verificar algumas exceções, em especial para os resultados relativos à pressão de $350 \mathrm{kPa}$. 


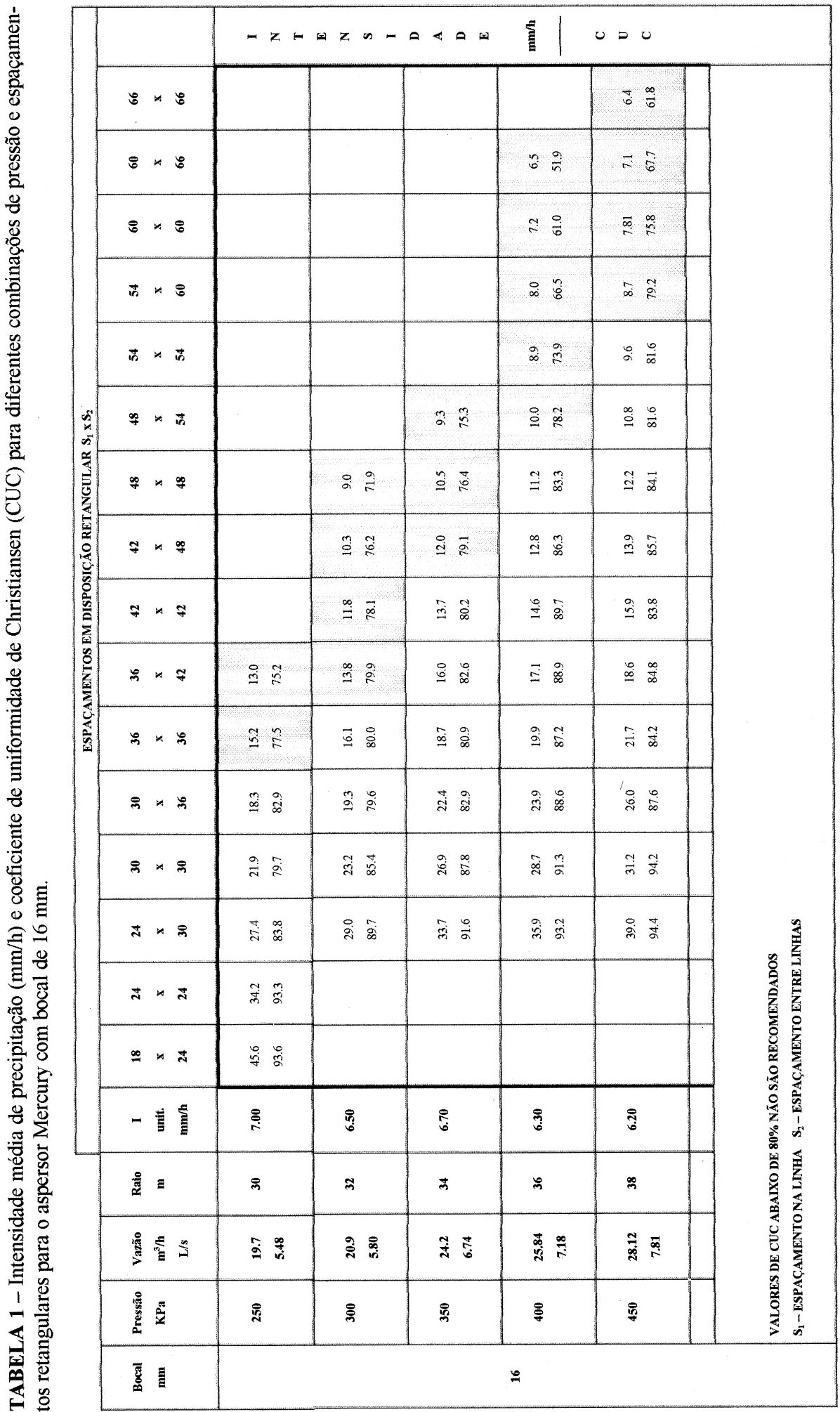




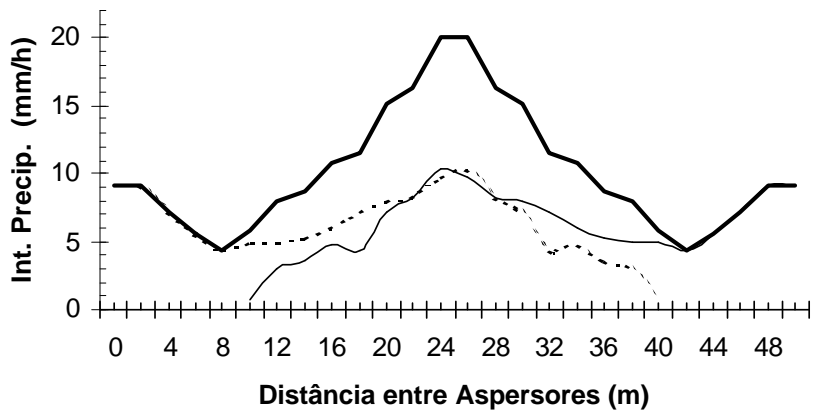

FIGURA 6 - Sobreposição para aspersores com perfil do tipo "doughnut", espaçados de 50 m.

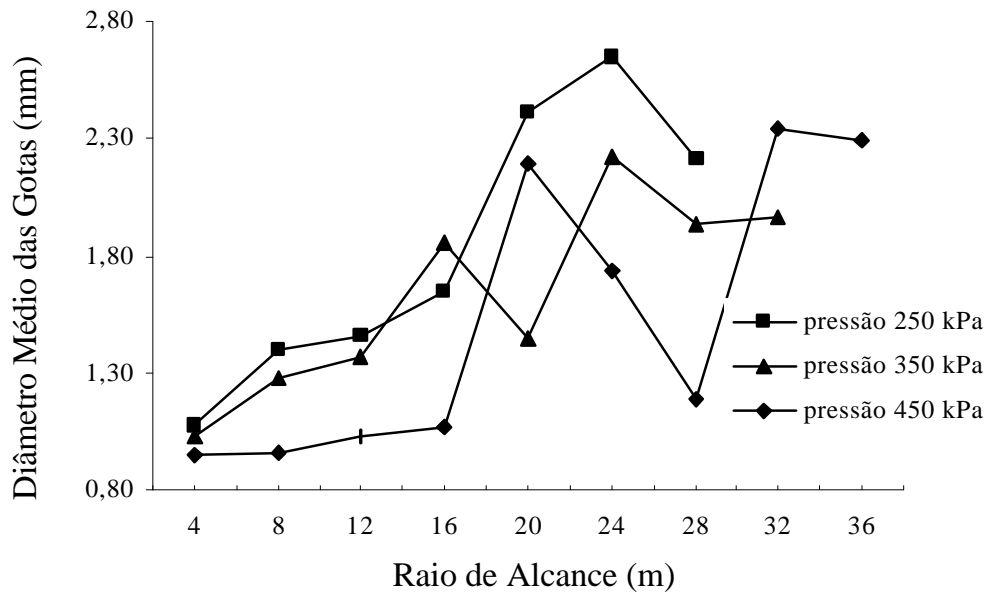

FIGURA 7 - Variação do diâmetro médio das gotas ao longo do raio de alcance do aspersor, para o bocal $16 \mathrm{~mm}$ e pressões de serviço 250,350 e $450 \mathrm{kPa}$.

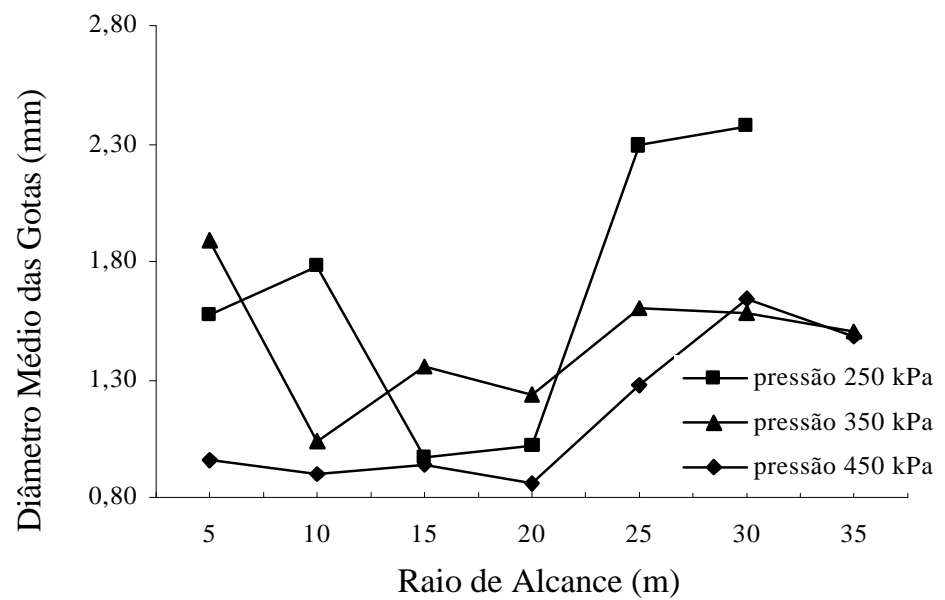

FIGURA 8 - Variação do diâmetro médio das gotas ao longo do raio de alcance do aspersor, para o bocal $20 \mathrm{~mm}$ e pressões de serviço 250,350 e $450 \mathrm{kPa}$.

Ciênc. agrotec., Lavras, v. 28, n. 4, p. 932-941, jul./ago., 2004 


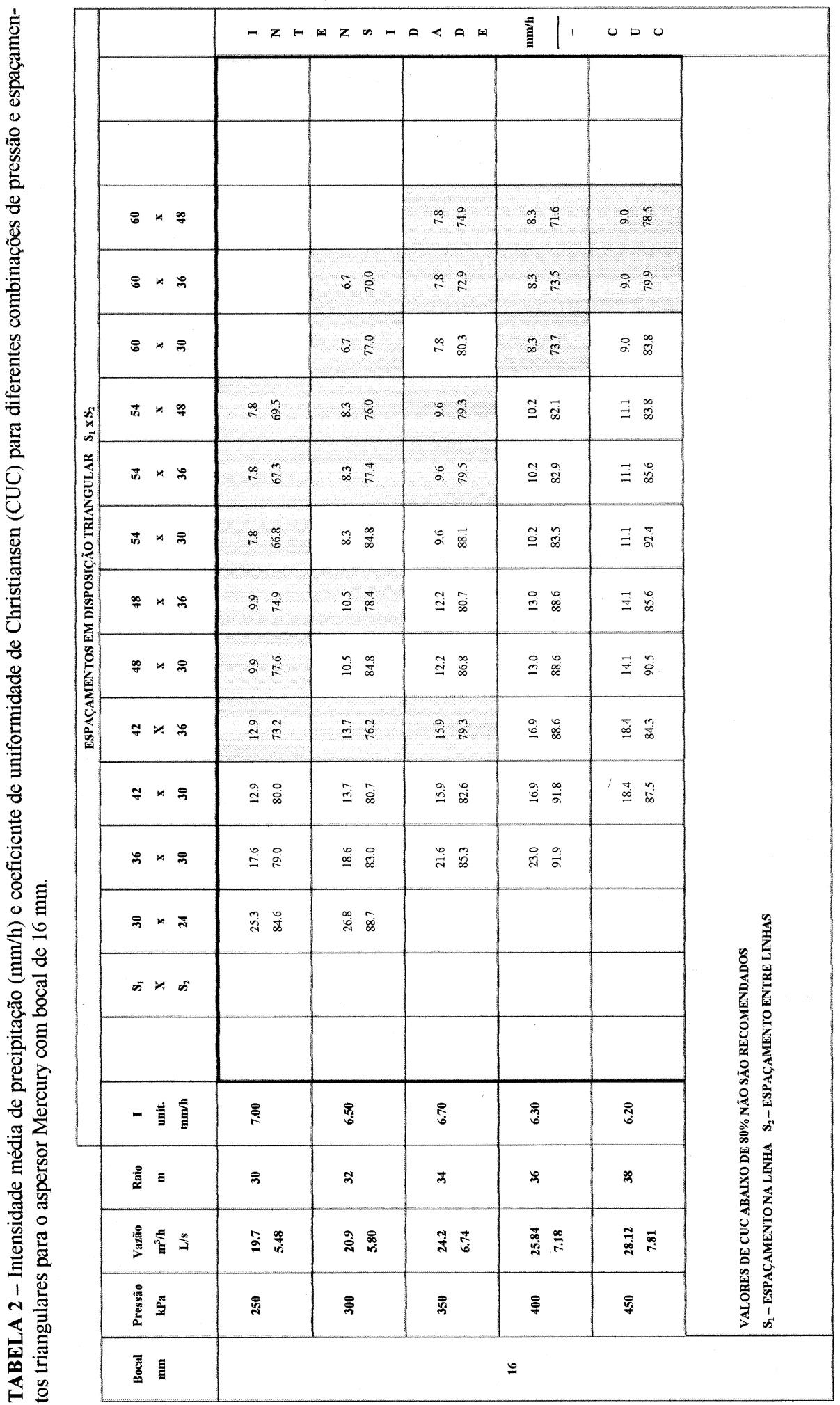




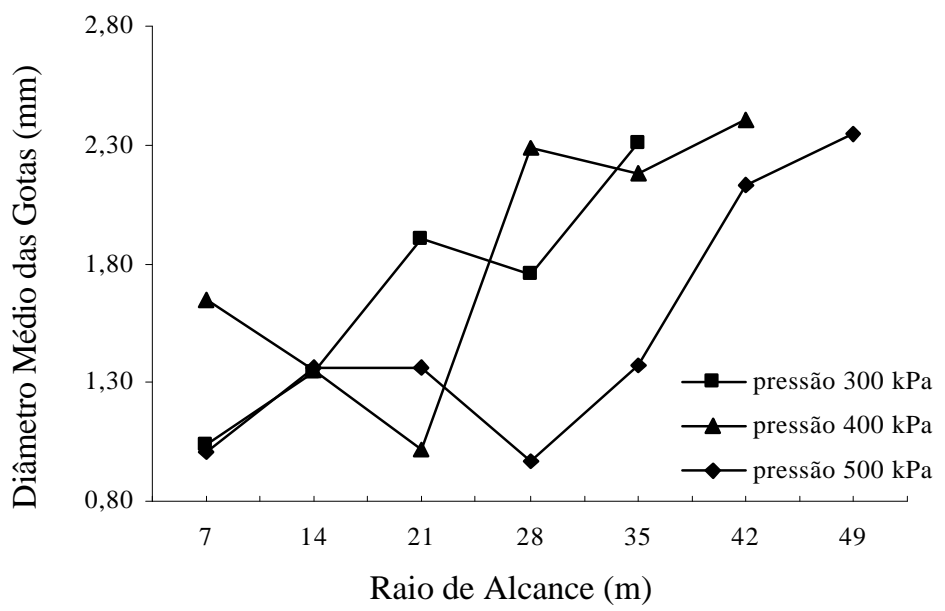

FIGURA 9 - Variação do diâmetro médio de gotas ao longo do raio de alcance do aspersor, para o bocal $22 \mathrm{~mm}$ e pressões de serviço 300, 400 e $500 \mathrm{kPa}$.

O comportamento-padrão estabelecido para os aspersores de que, com o aumento do raio molhado, tem-se um crescimento do diâmetro médio das gotas, não pode ser aplicado para aspersores de médio porte, em que constatou-se a existência de vários diâmetros de gotas ao longo do raio molhado, caracterizando um comportamento sem uniformidade, o que também foi constatado por Matsura (1993). Assim, com base nas informações obtidas neste trabalho, conclui-se que:

a) $\mathrm{O}$ diâmetro médio das gotas aumentou à medida que se reduziu a pressão de serviço, registrandose, entretanto, algumas exceções;

b) A relação vazão versus pressão foi bem representada pela equação potencial;

c) Existe uma predominância de formação do perfil de distribuição do tipo "doughnut" e retangular;

d) O sistema de rotação do tipo turbina apresentou boa eficiência, sendo o mesmo apenas influenciado pela pressão de serviço, garantindo, assim, uma melhor distribuição por quadrantes;

e) Recomenda-se a substituição do catálogo do fabricante pelas tabelas apresentadas neste trabalho, pois além dos valores de intensidade média de precipitação $(\mathrm{mm} / \mathrm{h})$ para os espaçamentos recomendados, apresentam os valores do coeficiente de uniformidade de Christiansen.

\section{AGRADECIMENTOS}

À Fundação de Amparo à Pesquisa do Estado de Minas Gerais - FAPEMIG - pela concessão da bolsa de
Mestrado ao primeiro autor, e à empresa Metal Lavras, pela cessão dos aspersores e da estrutura necessária aos testes.

\section{REFERÊNCIAS BIBLIOGRÁFICAS}

BERNARDO, S. Manual de irrigação. 6. ed. Viçosa: UFV, 1995. 596 p.

CARVAlHO, J. A. Tamanho de gotas e uniformidade de distribuição de água de dois aspersores de média pressão. 1991. 73 f. Dissertação (Mestrado) - Universidade Federal de Lavras, Lavras, 1991.

GOMES, H. P. Engenharia de Irrigação: hidráulica dos sistemas pressurizados, aspersão e gotejamento. João Pessoa: UFPB, 1994. 344 p.

INTERNATIONAL STANDARD ORGANIZATION. Agricultural irrigation equipament: rotating sprinklers: part 1: design and operational requirements. ISO 7749-1. Genève, 1995. 12 p.

INTERNATIONAL STANDARD ORGANIZATION. Agricultural irrigation equipament: rotating sprinklers: part 2: uniformity of distribu and test methods. ISO 7749-2. Genève, 1990. 8 p.

KELLER, J.; BLIESNER, R. D. Sprinkler and trickle irrigation. New York: Van Nostrand Reinhold, 1990. $652 \mathrm{p}$. 
MARTÍN-BENITO, J. M. T.; GÓMEZ, M. V.; PARDO, J. L. Working conditions of sprinkler to optimize application of water. Journal of Irrigation and Drainage Engineering, New York, v. 118, n. 6, p. 895-914, 1992.

MATSURA, E. E. Granulometria de gotas de um aspersor tipo canhão. In: CONGRESSO BRASILEIRO DE ENGENHARIA AGRÍCOLA, 12., 1993, Ilhéus. Anais... Ilhéus: SBEA/CEPLAC, 1993. v. 5, p. 28402846.

ROCHA, F. A. Desempenho de um equipamento de irrigação autopropelido em condições de campo. 2000. 80 p. Dissertação (Mestrado) - Universidade Federal de Lavras, Lavras, 2000.
ROCHA, W. W. Influência de ângulos setoriais e pressão de serviço na uniformidade de distribuição de água de um equipamento de irrigação autopropelido. 1998. 46 p. Dissertação (Mestrado) - Universidade Federal de Lavras, Lavras, 1998.

TOMAZELA, C. Otimização do uso de um aspersor tipo canhão em função das características operacionais e velocidade média do vento. 1996. $59 \mathrm{f}$. Tese (Doutorado) - Escola Superior de Agricultura Luiz de Queiroz, Piracicaba, 1996.

VILELA, L. A. Avaliação do impacto de gotas sobre a superfície de um latossolo vermelho escuro irrigado por aspersão. 1995. 76 f. Dissertação (Mestrado) Universidade Federal de Lavras, Lavras, 1995. 